\title{
Equilíbrio postural em idosos com e sem Diabetes Mellitus tipo 2: Uma análise comparativa
}

\author{
Postural balance in the elderly with and without Type 2 Diabetes Mellitus: A comparative analysis \\ Equilibrio postural en ancianos con y sin Diabetes Mellitus tipo 2: un análisis comparativo
}

Recebido: 26/02/2021 | Revisado: 07/03/2021 | Aceito: 08/03/2021 | Publicado: 16/03/2021

\author{
Vanessa da Nóbrega Dias \\ ORCID: https://orcid.org/0000-0003-4064-7207 \\ Universidade Federal do Rio Grande do Norte, Brasil \\ E-mail: vanessanobrega.d@hotmail.com \\ Maria Clara Almeida Jacome \\ ORCID: https://orcid.org/0000-0002-4651-3560 \\ Universidade Federal do Rios Grande do Norte, Brasil \\ E-mail: clara.jacome@ hotmail.com \\ Wesley Ribeiro Costa Meneses \\ ORCID: https://orcid.org/0000-0002-7688-7248 \\ Universidade Federal do Rio Grande do Norte, Brasil \\ E-mail:wrcmenezes123@gmail.com \\ Adriana Guedes Carlos \\ ORCID: https://orcid.org/0000-0003-0747-5044 \\ Universidade Federal do Rio Grande do Norte, Brasil \\ E-mail: adrianaguedes@ hotmail.com \\ Juliana Maria Gazzola \\ ORCID: https://orcid.org/0000-0002-9333-1831 \\ Universidade Federal do Rio Grande do Norte, Brasil \\ E-mail: juliana.gazzola@ terra.com.br
}

\begin{abstract}
Resumo
O presente estudo teve como objetivo investigar o equilíbrio postural das pessoas idosas com e sem Diabetes Mellitus tipo 2 (DM2) e verificar as características clínicas associadas. Trata-se de um estudo observacional, analítico e transversal; avaliou-se 233 pessoas idosas (60 anos ou mais), sendo 147 com diagnóstico de DM2 (G1) e 86 sem DM2 (G2). O equilíbrio postural foi avaliado por meio do Mini - The Balance Evaluation Systems Test (MiniBESTest). As associações entre os escores totais do G1 e G2 foram verificadas por meio dos testes de Mann-Whitney e teste QuiQuadrado, $\alpha=0,05$. Após a análise inferencial, foi observada significância entre as variáveis dos grupos G1 e G2: IMC $(\mathrm{p}=0,048)$, número de medicamentos $(\mathrm{p}=0,001)$, TUGt motora $(\mathrm{p}=0,015)$, FPP $(\mathrm{p}<0,001)$, WHODAS $(\mathrm{p}=0,011)$, EDG $(\mathrm{p}=0,001)$, presença de alterações nos pés $(\mathrm{p}<0,001)$, calosidade $(\mathrm{p}<0,001)$, alterações articulares $(\mathrm{p}<0,001)$, alterações dermatológicas $(p<0,001)$, sensibilidade vibratória $(p<0,001)$, sensibilidade cutâneo-protetora $(p<0,001)$, hipotensão ortostática $(\mathrm{p}=0,003)$ e medo de quedas $(\mathrm{p}=0,002)$. A média do MiniBesTest foi de 22,15 $\pm 4,64$ pontos para o grupo com DM2 e 21,94 \pm 4,89 pontos para o grupo sem DM2. Foi observado também, significância nos domínios do Mini BESTest: ajustes antecipatórios $(\mathrm{p}=0,001)$ e respostas posturais $(\mathrm{p}=0,042)$ em idosos com e sem DM2. Os idosos do G1 apresentam maior prejuízo do equilíbrio postural, quando associados à presença de sintomas depressivos, diminuição da mobilidade, presença de comorbidades, polifarmácia, alterações nos pés e medo de quedas.

Palavras-chave: Equilíbrio postural; Idoso; Diabetes mellitus; Complicações do diabetes.
\end{abstract}

\begin{abstract}
The present study aimed to investigate the postural balance of elderly people with and without Type 2 Diabetes Mellitus (DM2) and to verify the associated clinical characteristics. It is an observational, analytical and crosssectional study; 233 elderly people (60 years or older) were evaluated, 147 with a diagnosis of DM2 (G1) and 86 without DM2 (G2). Postural balance was assessed using the Mini - The Balance Evaluation Systems Test (MiniBESTest). The associations between the total scores of G1 and G2 were verified using the Mann-Whitney tests and the Chi-square test, $\alpha=0.05$. After inferential analysis, significance was observed between variables in groups G1 and G2: BMI ( $p=0.048)$, number of medications $(\mathrm{p}=0.001)$, motor TUGt $(\mathrm{p}=0.015)$, FPP $(\mathrm{p}<0.001)$, WHODAS ( $p=0.011)$, EDG $(p=0.001)$, presence of changes in the feet $(p<0.001)$, calluses $(p<0.001)$, joint changes $(p<0.001)$, dermatological changes $(p<0.001)$, vibratory sensitivity $(p<0.001)$, skin-protective sensitivity $(p<0.001)$, orthostatic hypotension $(\mathrm{p}=0.003)$ and fear of falls $(\mathrm{p}=0.002)$. The MiniBesTest average was $22.15 \pm 4.64$ points for the group with DM2 and $21.94 \pm 4.89$ points for the group without DM2. Significance was also observed in the Mini BESTest domains: anticipatory adjustments $(\mathrm{p}=0.001)$ and postural responses $(\mathrm{p}=0.042)$ in the elderly with and without
\end{abstract}


DM2. G1 elderly people have greater impairment of postural balance when associated with the presence of depressive symptoms, decreased mobility, the presence of comorbidities, polypharmacy, changes in the feet and fear of falling.

Keywords: Postural balance; Aged; Diabetes mellitus, Diabetes complications.

\section{Resumen}

El presente estudio tuvo como objetivo investigar el equilibrio postural de personas mayores con y sin diabetes mellitus tipo 2 (DM2) y verificar las características clínicas asociadas. Es un estudio observacional, analítico y transversal; Se evaluaron 233 ancianos (60 años o más), 147 con diagnóstico de DM2 (G1) y 86 sin DM2 (G2). El equilibrio postural se evaluó utilizando el Mini - The Balance Evaluation Systems Test (MiniBESTest). Las asociaciones entre los puntajes totales de G1 y G2 se verificaron utilizando las pruebas de Mann-Whitney y la prueba de Chi-cuadrado, $\alpha=0.05$. Tras el análisis inferencial se observó significación entre las variables de los grupos G1 y G2: IMC ( $\mathrm{p}=0,048)$, número de medicamentos $(\mathrm{p}=0,001)$, TUGt motor $(\mathrm{p}=0,015)$, FPP $(\mathrm{p}<0,001)$, WHODAS ( $\mathrm{p}$ $=0,011)$, EDG $(p=0,001)$, presencia de cambios en los pies $(\mathrm{p}<0,001)$, callosidades $(\mathrm{p}<0,001)$, cambios articulares ( $p<0,001)$, cambios dermatológicos $(p<0,001)$, sensibilidad vibratoria $(p<0,001)$, sensibilidad protectora de la piel ( $\mathrm{p}<0,001)$, hipotensión ortostática $(\mathrm{p}=0,003)$ y miedo a las caídas $(\mathrm{p}=0,002)$. La media del MiniBesTest fue de $22,15 \pm 4,64$ puntos para el grupo con DM2 y de 21,94 \pm 4,89 puntos para el grupo sin DM2. También se observó significancia en los dominios Mini BESTest: ajustes anticipatorios $(p=0,001)$ y respuestas posturales $(p=0,042)$ en ancianos con y sin DM2. Los ancianos G1 tienen mayor deterioro del equilibrio postural cuando se asocia a la presencia de síntomas depresivos, disminución de la movilidad, presencia de comorbilidades, polifarmacia, alteraciones en los pies y miedo a caer.

Palabras clave: Equilíbrio postural; Anciano; Diabetes mellitus; Complicaciones de la diabetes.

\section{Introdução}

No Brasil, a população com idade superior ou igual a 60 anos chega a quase 15 milhões de pessoas $(8,6 \%$ da população brasileira) (Chau, et al., 2013). Esse aumento da expectativa de vida vem acompanhado pelos acometimentos comuns da idade com o predomínio de doenças crônicas, como o Diabetes Mellitus tipo 2 (DM2) (Horak, et al., 2009). Tanto o envelhecimento quanto o diabetes são importantes fatores de risco para o declínio funcional e a diminuição da qualidade de vida, além de gerar maiores gastos para o sistema de saúde (Lima Filho, et al., 2020; Menezes, et al., 2014).

As complicações comumente associadas ao DM2 são as doenças vasculares, renais, do sistema nervoso e da visão, diminuição da massa muscular, presença de comorbidades, queixa de dor, sintomas depressivos e polifarmácia (Mettelinge, et al., 2013), estas alterações contribuem para o déficit do equilíbrio postural e aumento do número de quedas (Noce, et al., 2019).

O controle postural requer a integração central de três sistemas sensoriais distintos: vestibulares, visuais e somatossensoriais. Os seres humanos utilizam com grande intensidade as informações dos membros inferiores para manter o equilíbrio corporal. Sendo assim, o dano nervoso associado a neuropatia periférica diabética (NPD) pode contribuir para alterações na marcha e déficits no equilíbrio (Petrofsk, et al., 2006; Rashedi, et al., 2019).

As evidências científicas comprovam a associação entre DM2 e déficit no equilíbrio corporal e, consequente, risco de queda em pacientes com complicações diabéticas, com foco na neuropatia periférica, porém, se a queda está associada a diversos fatores, que são agravados pelas complicações da DM2, então, indivíduos sem complicações podem apresentar menos chance de cair ou o risco de cair permanece o mesmo e está associado também a outras variáveis? Nesse sentido, este estudo objetivou investigar os fatores associados as alterações do equilíbrio postural em pessoas idosas com e sem diabetes.

\section{Metodologia}

Trata-se de um estudo do tipo observacional, quantitativo, analítico de caráter transversal (Pereira, et al., 2018). cuja amostra foi constituída por 233 idosos com 60 anos ou mais, com e sem diagnóstico de Diabetes Mellitus tipo 2, segundo os critérios da American Diabetes Association (ADA), de ambos os sexos e que aceitem participar lendo e assinando o Termo de Consentimento Livre e Esclarecido (TCLE). A amostra foi dividida em dois grupos: grupo 1 (G1): com diagnóstico de DM tipo $2(\mathrm{~N}=147)$ e grupo $2(\mathrm{G} 2)$ : sem DM tipo $2(\mathrm{~N}=86)$. 
Foram excluídos do estudo os idosos que apresentaram limitações físicas e cognitivas que impedissem a realização dos testes de equilíbrio, como incapacidade de compreender e atender a comando verbal simples e/ou imitar movimentos; aqueles que apresentaram acuidades visual e auditiva gravemente diminuídas e absolutamente incapacitantes às atividades de vida diária, mesmo com uso de lentes corretivas e/ou aparelhos de amplificação sonora; aqueles que apresentaram amputações de membros inferiores e de membros superiores acima do nível das articulações metatarso-falangeanas e metacarpofalangeanas, respectivamente; aqueles em uso de próteses em membros inferiores e de membros superiores; aqueles impossibilitados de deambular independentemente e com locomoção exclusivamente por cadeira de rodas. Também foram excluídos os pacientes que já realizaram alguma forma de reabilitação do equilíbrio corporal nos últimos seis meses anteriores à avaliação.

Cada idoso recebeu informações sobre os objetivos e os procedimentos básicos da pesquisa; aqueles que decidiram participar do estudo serão agendados para o preenchimento do protocolo e assinaram o TCLE. A avaliação ambulatorial foi realizada no Núcleo Avançado de Pesquisa e Inovação Tecnológica em Saúde (NAPS) do Hospital Universitário Onofre Lopes na Universidade Federal do Rio Grande do Norte, realizada por avaliadores previamente treinados e após a aprovação do Comitê de Ética em pesquisa do HUOL, sob protocolo nº. 38152719.1.0000.5537.

Os dados clínicos avaliados foram: Altura, peso e Índice de Massa Corporal (IMC), realização de atividade física regular, o número de doenças, o número de medicamentos utilizados, uso de dispositivo de auxílio à marcha, inspeção dos pés, presença de hipotensão ortostática, presença de dores em membros inferiores e sua intensidade, testes de sensibilidade vibratória, proprioceptiva e cutânea protetora, ocorrência de queda a circunstâncias desse acontecimento e a presença de tontura.

Os pés foram inspecionados em decúbito dorsal, ou seja, exame realizado na ausência de carga sobre as estruturas desse segmento, a fim de verificar se há presença das principais deformidades articulares e alterações dermatológicas, tais como: hálux valgo, varo, sobreposição de dedos, dedos em garra, em martelo, úlceras, alterações tróficas da pele, condições de hidratação da pele, hiperqueratoses, alterações ungueais, alterações de coloração da pele, dentre outras.

A hipotensão ortostática foi mensurada averiguando a pressão arterial com o paciente deitado e em pé; sabe-se que a queda de $20 \mathrm{~mm} \mathrm{Hg}$ da pressão arterial sistólica e $10 \mathrm{mmHg}$ da pressão arterial diastólica quando este paciente fica em pé e ainda sintomas de hipoperfusão cerebral caracteriza este problema (Silveira, 2013). Também foi questionado quanto à presença de dores em membros inferiores e à intensidade da dor, por meio da Escala Visual Analógica.

Para sensibilidade vibratória, foi utilizado diapasão de $128 \mathrm{~Hz}$ nos maléolos medial e lateral e base do primeiro metatarso, face dorsal, bilateralmente. Na presença de úlceras, a mesma conduta anterior foi eleita.

O teste de sensibilidade proprioceptiva ou cinético postural foi realizado com o paciente em decúbito dorsal. O paciente deve manter-se com os olhos fechados, e então o examinador posiciona um dos membros em determinada posição e solicita para que o paciente fique o mesmo posicionamento do membro contralateral. Se houver integridade da sensibilidade profunda o paciente conseguirá realizar o movimento corretamente (Sociedade Brasileira de Diabetes, 2013).

Para avaliação funcional do equilíbrio corporal foi utilizado o Mini - The Balance Evaluation Systems Test (BESTest) MiniBESTest. O MiniBESTest é formado por 14 testes, com um total de 36 itens. As questões são divididas em quatro seções, que correspondem aos sistemas responsáveis pela manutenção do equilíbrio, são elas: 1. Transições e ajustes posturais antecipatórios; 2. Respostas posturais à perturbação; 3. Orientação sensorial; e 4. Estabilidade na marcha. Cada item é pontuado em uma escala ordinal de três pontos variando de zero a dois pontos, em que zero é o pior desempenho e dois, o melhor (Speciali, 2020). 
A pontuação sugerida do Mini BESTest para identificar idosos com histórico de quedas é de 16 em 28, o que dá uma sensibilidade de $85 \%$ e uma especificidade de $75 \%$. Para a avaliação do risco de quedas, foi considerado um tempo de 16 segundos para a pontuação total do MiniBest, ou seja, o maior ponto de corte de 16 indica baixo risco de queda risco de queda (Strain, 2018).

A avaliação da mobilidade funcional foi feita através do Timed Up and Go Test (TUGt) (Aveiro, 2012) e para a funcionalidade foi utilizado o World Health Organization Disability Assessment Schedule (WHODAS 2.0) (Carlozzi, et al., 2015). Os dados psico-cognitivos foram avaliados através do teste cognitivo Mini-Mental State Exam (MMSE) (Folstein, et al., 1975) e da Escala de Depressão Geriátrica Abreviada (EDG) (Yesavage, 1983).

A normalidade de distribuição dos dados foi verificada por meio do teste Kolmogorov-Smirnoff. Foram inicialmente realizadas análises descritivas simples. Para análise inferencial, foram verificadas associações entre o escore total do G1 e G2 por meio dos testes de Mann-Whitney para variáveis escalares, e teste Qui-Quadrado ou Teste de Fisher para variáveis categóricas. Para todas as análises estatísticas foi adotado um nível de significância de 5\% ( $<<0,05)$. Todos os procedimentos estatísticos foram realizados por meio do software SPSS versão 20.0 para Windows.

\section{Resultados}

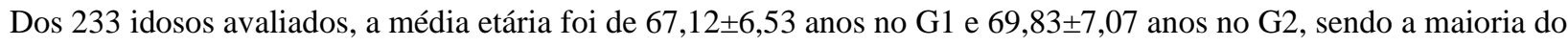
sexo feminino (68,8\%), com vida conjugal (60,3\%) e com nível de escolaridade até primário incompleto (42,3\%). A média do IMC foi de $28,53 \pm 4,50 \mathrm{Kg} / \mathrm{m}^{2}$ para G1 e $26,63 \pm 4,25 \mathrm{Kg} / \mathrm{m}^{2}$ para G2, caracterizando a amostra como sobrepeso.

A maioria dos idosos diabéticos apresentou entre cinco doenças diagnosticadas ou mais $(83,7 \%)$, enquanto que no grupo de não diabéticos a maioria apresentou duas ou quatro doenças diagnosticadas (55,8\%); a utilização de dois ou quatro medicamentos apresentou maioria em ambos nos grupos (73,6\% para G1 e 64,0\% para G2); quanto a presença de uma ou mais alteração nos pés (G1 apresentou 83,1\% de uma ou mais alterações e G2 apresentou 96,5\% sem alterações, já com relação ao medo de cair a maioria relatou que tinha (78,1\% para G1 e 63,5\% para G2).

A análise inferencial das variáveis do estudo pode ser encontrada na Tabela 1. Nela, é possível observar a diferença entre idosos diabéticos e não diabéticos e assim, entender os fatores de risco agravantes para a população idosa acometida pelo Diabetes Mellitus tipo 2.

Tabela 1. Análise inferencial entre a variável idosos com e sem DM2 e as variáveis clínico-funcionais e psicocognitivas de uma amostra de 233 idosos com Diabetes Mellitus tipo 2 avaliados em um hospital universitário do nordeste brasileiro.

\begin{tabular}{|c|c|c|c|c|c|}
\hline \multirow{3}{*}{ Variáveis } & \multirow{2}{*}{\multicolumn{2}{|c|}{$\begin{array}{l}\text { Idosos com DM2 } \\
\text { (G1) }\end{array}$}} & \multirow{2}{*}{\multicolumn{2}{|c|}{$\begin{array}{l}\text { Idosos sem DM2 } \\
\text { (G2) }\end{array}$}} & \multirow{3}{*}{ P valor } \\
\hline & & & & & \\
\hline & $\mathbf{N}$ & $\%$ & $\mathbf{N}$ & $\%$ & \\
\hline \multicolumn{6}{|l|}{$\mathbf{N}^{\circ}$ de doenças } \\
\hline Sem doenças & 01 & $0,7 \%$ & 13 & $15,1 \%$ & \multirow{3}{*}{$p<0,001$} \\
\hline Uma ou duas & 23 & $15,6 \%$ & 44 & $51,2 \%$ & \\
\hline Três ou mais & 123 & $83,7 \%$ & 29 & $33,7 \%$ & \\
\hline
\end{tabular}


Research, Society and Development, v. 10, n. 3, e29610313345, 2021

(CC BY 4.0) | ISSN 2525-3409 | DOI: http://dx.doi.org/10.33448/rsd-v10i3.13345

$\mathrm{N}^{\mathrm{o}}$ de medicamentos

Não usa

Um ou dois

Três ou mais

\section{Dor em MMII}

Sim

Não

69

131

$0,7 \%$

$9,7 \%$

Dispositivo de auxílio à marcha

Não utiliza

140

Utiliza

Alterações nos pés

Sem alterações

Uma a três alterações

Quatro ou mais alterações

Presença de calosidade nos pés

Sim

Não

Deformidades Articulares

Sim

Não

\section{Alterações Dermatológicas}

Sim

118

Não

Sensibilidade vibratória

Alterada

57

Preservada

55
$89,6 \%$

$53,1 \%$

$46,9 \%$

40

$53,5 \%$

$46,5 \%$

$\mathrm{p}=0,950$

$95,2 \%$

$4,8 \%$
$16,3 \%$

$40,8 \%$

$42,9 \%$

$49,7 \%$

$50,3 \%$

$37,4 \%$

$62,6 \%$

$80,3 \%$

$19,7 \%$

83

$3,5 \%$

$96,5 \%$

$96,5 \%$

$2,3 \%$

$1,2 \%$

$3,5 \%$

$96,5 \%$

$97,7 \%$ $p=0,001$

$38,4 \%$

$96,5 \%$

$p=0,643$

$3,5 \%$

p $<0,001$

p $<0,001$

p<0,001

p<0,001

$61,2 \%$

7

$91,9 \%$

$8,1 \%$

p<0,001 
Sensibilidade cutâneo protetora

Alterada

Preservada

\section{Hipotensão ortostática}

$\operatorname{Sim}$

Não

\section{Tontura}

$\operatorname{Sim}$

Não

\section{Medo de quedas}

$\operatorname{Sim}$

Não

\section{Tendência a quedas}

Sim

Não

76

70

$52,1 \%$

$47,9 \%$

$21,9 \%$

54

31

33

53

74

$14,0 \%$

$86,0 \%$

$\mathbf{p}=\mathbf{0 , 0 3 3}$

$38,4 \%$

$61,6 \%$

$\mathrm{p}=0,282$

$36,5 \%$

$55,3 \%$

$44,7 \%$

$\mathbf{p}<0,001$

\section{MMSE}

Déficit cognitivo

Sem déficit cognitivo

64

83

\section{Escala de Depressão Geriátrica}

Com sintomas depressivos

Sem sintomas depressivos
73

73
$43,2 \%$

$56,8 \%$

$50,0 \%$

$50,0 \%$
47

38

46

40

22

62
$52,9 \%$

$47,1 \%$ 
Foi observado também, significância nos domínios do Mini BESTest: ajustes antecipatórios ( $\mathrm{p}=0,001)$ e respostas posturais $(\mathrm{p}=0,042)$ em idosos com e sem DM2, conforme descritos na tabela 2 . A curva ROC indicou que a pontuação que delimita melhor o grupo com DM2 é de $\geq 23$ pontos ( $\mathrm{AUC}=0,50)$.

A Tabela 2 demonstra qual domínio do Mini BesTest foi pior acometido pelo grupo de idosos com Diabetes Mellitus tipo 2.

Tabela 2. Dados da análise inferencial do Mini BesTest de uma amostra de 233 idosos entre a variável idosos com e sem DM2 avaliados em um hospital do nordeste brasileiro.

Variáveis

\begin{tabular}{|c|c|c|c|c|c|}
\hline & \multicolumn{2}{|c|}{$\operatorname{Sim}(N=147)$} & \multicolumn{2}{|c|}{ Não (N=86) } & \\
\hline & Mediana & IC $(95 \%)$ & Mediana & IC $(95 \%)$ & \\
\hline Mini BESTest total & 82,1 & $76,5-81,9$ & 78,2 & $74,5-81,9$ & 0,644 \\
\hline $\begin{array}{l}\text { Ajustes Posturais } \\
\text { Antecipatórios }\end{array}$ & 83,3 & $74,1-80,0$ & 67,0 & $63,1-72,9$ & $<0,001$ \\
\hline Respostas Posturais & 66,6 & $56,6-67,1$ & 72,4 & $65,5-79,4$ & $\mathbf{0 , 0 3 5}$ \\
\hline Orientação Sensorial & 100,0 & $89,6-94,7$ & 100,0 & $86,1-93,5$ & 0,128 \\
\hline $\begin{array}{c}\text { Estabilidade Durante a } \\
\text { Marcha }\end{array}$ & 90,0 & $79,9-86,2$ & 90,0 & $76,6-85,4$ & 0,390 \\
\hline
\end{tabular}

Fonte: Autores (2021).

\section{Discussão}

Nesse estudo foram verificadas alterações no equilíbrio postural de idosos com diabetes mellitus tipo 2 (DM2) quando comparado aos idosos sem diabetes. Esse fato foi evidenciado por uma maior presença de doenças diagnosticadas, alterações no índice de massa corporal, maior presença de alterações nos pés e perda de sensibilidade. Essas alterações ocorrem, pois, a DM2 gera repercussões sistêmicas de forma negativa no organismo dos indivíduos portadores, como o surgimento de outras doenças, como a obesidade, polineuropatias e as doenças arteriais periféricas. Todos esses distúrbios alteram o equilíbrio e a marcha, tornando o idoso mais vulnerável a quedas (Chau, et al., 2013).

Foi constatado que um IMC mais alto nos pacientes idosos diabéticos quando comparado aos não diabéticos, o que já se é esperado uma vez que à medida que o indivíduo aumenta sua massa gorda, seus níveis glicêmicos também se elevam, fato este relacionado a um aumento da resistência à insulina, aumentando o risco de desenvolvimento do DM2 (Horak, et al., 2009).

Ficou evidenciado também, que os idosos diabéticos fazem uso de uma maior quantidade de medicamentos quando comparado aos não diabéticos. É consensual que a diabetes gera o surgimento de comorbidades desde vasculares a neurossensoriais, explicando assim o motivo pelo qual o tratamento medicamentoso se torna mais significativo nesses pacientes (Vinik, et al., 2017).

Apesar de na análise do Mini BESTest não ter havido diferença significativa entre os dois grupos, idosos com DM2 apresentaram menor pontuação nos domínios específicos que avaliam equilíbrio (ajustes antecipatórios e respostas posturais) em relação aos idosos sem a mesma doença, o que sugere um maior risco de queda. Esses achados se justificam pelo fato de os pacientes com diabetes apresentarem distúrbios associados, como por exemplo a neuropatia periférica, que é causada pelo controle inadequado da glicose. A neuropatia periférica faz com que os nervos motores e sensoriais não trabalhem 
corretamente, causando perda de força e perda de sensibilidade nas extremidades, e consequentemente, diminuição da função e perda do equilíbrio (Wanjgarten, et al., 2017).

Além disso, idosos com DM2 também obtiveram maior expressividade de sintomas depressivos e apresentaram uma maior prevalência do medo de cair e, que podem ser preditores de marcha deficitária, pois a depressão pode causar diversos fatores como indiferença ao meio ambiente, adulteração do nível da atenção, redução do comprimento da passada, diminuição da energia, diminuição da autoconfiança, reclusão, inatividade e perdas cognitivas. Portanto, nesse estudo, ser idoso com DM2 resultou em um maior declínio do equilíbrio postural e, consequentemente, na marcha do que em idosos sem o mesmo tipo de diabetes (Yingyongyudha, 2015).

\section{Conclusão}

Os idosos do G1 apresentaram maior prejuízo do equilíbrio postural quando associados a sobrepeso, maior presença de doenças diagnosticadas e maior número de medicamentos em uso, alterações nos pés e na sensibilidade, medo de quedas e presença de sintomas depressivos. Esses aspectos físicos e cognitivos foram significativamente piores em idosos com DM2 quando comparado a idosos sem diabetes.

Os achados mostram a necessidade de intervenção precoce nessa população, com estratégias de prevenção para minimizar as alterações de marcha e equilíbrio, e consequentemente o risco de quedas em programas de educação em DM2. Diminuindo, assim, os gastos em saúde pública em consequências de quedas, bem como a mortalidade e morbidade.

Sendo assim, sugere-se a importância da realização de estudos longitudinais que avaliem com mais precisão o impacto do DM 2 sobre os sistemas corporais e suas consequências para o equilíbrio corporal.

\section{Referências}

Aveiro, M. C., Driusso, P., Barham, E. J., Pavarini, S. C. I., \& Oishi, J. (2012). Mobilidade e risco de quedas de população idosa da comunidade de São Carlos. Ciênc. saúde coletiva, 17(9), 2481-2488.

Carlozzi, N. E., Kratz, A. L., Downing, N. R., Goodnight, S., Miner, Migliore, N., \& Paulsen, J. S. (2015). Validity of the 12-item World Health Organization Disability Assessment Schedule 2.0 (WHODAS 2.0) in individuals with Huntington disease (HD), Qual Life Res., $24(8), 1963-71$.

Chau, R. M. W., Ng, T. K. W. Kwan, Choi, C-H., \& Cheing, G. L. Y. Risk of fall for people with diabetes. (2013). Disability and rehabilitation, 35(23), 19751980 .

Folstein, F. F., Folstein, S. E., \& Mchugh, P. R. (1975). Mini-mental state: a practical method for grading the cognitive states for the clinician. J Psychiatr Res., 12(3), 189-198.

Horak, B. F., Wrisley, M. D., \& James, F. (2009). The Balance Evaluation Systems Test (BESTest) to differentiate balance deficits. Physical therapy, 89(5), 489-498.

Lima Filho, B. F., Gama, A.G.D., Dias, V. N., Silva, E. M. T., Cavalcanti, F. A. C., \& Gazzola, J. M. The frailty syndrome in older adults with type 2 diabetes mellitus and associated factors. (2020). Rev. bras. geriatr. gerontol, 23(1), e190196.

Menezes, T. N., Sousa, N. D. S., Moreira, A.S., Pedraza, D. F. (2014). Diabetes mellitus referido e fatores associados em idosos residentes em Campina Grande, Paraíba. Rev. bras. geriatr. gerontol. 17(4), 829-839.

Mettelinge, R. T., Dirk, C., Calders, P., Noortgate, V. D. N., \& Delbaere, K. (2013). Understanding the Relationship between Type 2 Diabetes Mellitus and Falls in Older Adults: A Prospective Cohort Study. PLoS ONE, 8(6), e67055.

Noce, K. R., Borem, I. L., Sampaio, R. F., Ferreira, V. K. G., Almeida, J. C., Guimarães, S. B. B., \& Moreira, B. S. (2019). Frailty status and gait parameters of older women with type 2 diabetes. Canadian Journal of Diabetes, 43(2), 121-127.

Pereira, A.S., Shitsuka, D. M., Parreira, F. J. P., Shitsuka, R. (2018). Metodologia da pesquisa científica. UFSM. https://repositorio.ufsm.br/bitstream/handle/1/15824/Lic_Computacao_Metodologia-Pesquisa-Cientifica.pdf?sequence=1.

Petrofsky, J. S., Cuneo, M., Lee, S., Johnson, E., \& Lohman, E. (2006). Correlation between gait and balance in people with and without Type 2 diabetes in normal and subdued light. Med Sci Monit, 12(7), 273-281.

Rashedi, V., Iranpour, A., Mohseni, M., \& Borhaninejad, V. Risk factors for fall in elderly with diabetes mellitus type 2. (2019). Diabetes \& Metabolic Syndrome: Clinical Research \& Reviews, 13(5), 2347- 2351. 
Research, Society and Development, v. 10, n. 3, e29610313345, 2021

(CC BY 4.0) | ISSN 2525-3409 | DOI: http://dx.doi.org/10.33448/rsd-v10i3.13345

Silveira, L. A. G. (2013). Correlação entre obesidade e diabetes tipo 2. Pós-graduação Latu-Sensu em Fisiologia do Exercício e Avaliação-Morfofuncional Universidade Gama Filho.

Sociedade Brasileira de Diabetes. Consenso brasileiro sobre diabetes 2002: diagnóstico e classificação do diabetes melito e tratamento do diabetes melito tipo 2. (2003). Rio de Janeiro: Diagraphic.

Speciali, J.G. Semiotécnica neurológica. (2020). Medicina, 29(1), 19-31.

Strain, W. D., Hope, S. V., Green, A., Kar, P., Valabhji, J., \& Sinclair, A. J. (2018). Type 2 diabetes mellitus in older people: a brief statement of key principles of modern day management including the assessment of frailty. A national collaborative stakeholder initiative. Diabet. Med., 35, 838-84.

Vinik, A. I., Vinik, E. J., Colberg, S. R., \& Steven, M. (2015). Falls Risk in Older Adults with Type 2 Diabetes. Clínics in Geriatric Medicine, 31(1), 89-99.

Wanjgarten, M., Serro-Azu, J. B., \& Goulart, M. L. (2017) Abordagem das hipotensões ortostática e pós-prandial. Rev Bras Hipertens, 14(1), $29-32$.

Yesavage, J. A., Brink, T. L., Rose, T. L., Lum, O., Huang, V., Adey, M., \& Leirer, V. O. (1983). Development and validation of a geriatric depression screening scale: a preliminary report. Journal of psychiatric research, 17(1), 37-49.

Yingyongyudha, A., Saengsirisuwan, V., Panichaporn, W., \& Boonsinsukh, R. (2015). The Mini-Balance Evaluation Systems Test (Mini-BESTest) Demonstrates Higher Accuracy in Identifying Older Adult Participants With History of Falls Than Do the BESTest, Berg Balance Scale, or Timed Up and Go Test. J Geriatr Phys Ther., 38,1-7. 